\title{
Modal Analysis of the Tubular Space Frame of a Formula Student Race Car
}

Attila Schweighardt, Balázs Vehovszky, Dániel Feszty

Audi Hungaria Faculty of Automotive Engineering, Department of Whole Vehicle Engineering, Széchenyi István University. Egyetem tér 1, H-9026 Győr. Hungary. E-mail: schweighardtattia@gmail.com, vehovszky.balazs@sze.hu, feszty.daniel@sze.hu

This document presents the characterization of the dynamic mechanical properties of a racecar's frame. First, it introduces the applicability of modal analysis, then the modal analysis of a lightweight vehicle chassis will be detailed, which is the focal point of this paper. This analysis was performed to determine some of the modal parameters, in order to reduce the noise of the vehicle, the probability of a component failure and to improve the comfort. The simulation part of the applied analysis was based on dynamic FEM (Finite Element Method). The measurement part of it was based on measuring the FRFs (Frequency Response Functions), with the help of accelerometers fixed at the nodes of the frame. The excitation signals were provided by a shaker connected to the chassis. In order to provide good quality results, the processing and evaluation of the simulated and measured data has to be done properly, which is discussed in detail. However, one dominant factor of a modal analysis is to find the optimal measurement setup. For this reason, the details of the measurement setup will be included. Hence one of the goals was to improve the coherence curves of the FRFs. Thanks to the presented techniques, the coherence curves managed to be improved and the results of the simulation and the measurement were found to be in good agreement.

Keywords: Acoustics, Modal Analysis, Eigenmode, Eigenfrequency, Coherence

\section{Introduction}

Vehicles are subjected to dynamic excitations, vibrations and highly varying loads, which are in various frequency ranges. These can originate from different sources, e.g. from the engine, drivetrain, road, wind or audio devices. Due to load, fatigue, noise, comfort, safety and even vehicle dynamics considerations, it is usually advisable to avoid certain frequency ranges so that intense vibrations around the resonance frequencies do not occur.

Using modal analysis, the vibrations can be reduced by avoiding or detuning excitations near the natural frequencies. This can be done through the modification of the structure or by selecting suitable excitation frequency range. If it is not possible to tune the sources - like changing the idle or maximum rotational frequency of the engine or selecting a different vehicle velocity, thus changing the road or wind excitation - then the structure has to be modified. There are different possibilities to tune the acoustical behaviour of a mechanism, e.g. by changing the used material, the geometry of the structure and by adding extra damping elements. In case of the material properties, the Young's modulus, the density, the damping and the Poisson's ratio have to be considered during a modal analysis. From these, the Young's modulus and the density are the dominant factors from eigenfre- quency and eigenmode points of view. While the influence of the damping is less dominant, in contrast with the vibration amplitude response, on which the damping has significant impact. For example damping determines the maximum acceleration, displacement and stress amplitudes. Poisson's ratio has negligible effect on the dynamic characteristics.

With the help of dynamic Finite Element Method (FEM) and acoustical measurements the structure can be optimized. With modal analysis the modal behaviour and the modal parameters can be determined such as the mode shapes, the corresponding natural frequencies and the frequency dependent modal damping. The analysed vehicle, which is a race car designed for one of the SAE (Society of Automotive Engineers) organized competitions, is expected to experience road excitations between 0 and $20 \mathrm{~Hz}$. Within this range, the most common road excitation is around $10 \mathrm{~Hz}$. This is lower than the predicted first global flexural natural frequency of the frame, therefore this excitation is not critical from acoustic point of view. However, the excitation range of the engine is critical. The idle rotational frequency of the engine is $3000 \mathrm{rpm}(50 \mathrm{~Hz})$ and the maximum frequency of the engine is about $9000 \mathrm{rpm}(150 \mathrm{~Hz})$. Unfortunately, this excitation range covers exactly the most critical frequency band considering the frame's first few eigenfrequencies. This can result fatigue, component failure, noise and vibration issues and lack of grip, and 
furthermore, it has a huge effect on the comfort of the driver. These were some examples, in which modal analysis may be used.

This paper focuses mostly on the usage of modal analysis for noise- and vibration reduction. According to the rules of the SAE series, two requirements have to be fulfilled regarding the sound pressure level. At idle the noise of the car has to be below $103 \mathrm{~dB}$, as well as at $6500 \mathrm{rpm}$ (equal to $108.33 \mathrm{~Hz}$ ) it has to be under $110 \mathrm{~dB}$, using $\mathrm{dB}(\mathrm{C})$ frequency filter [1]. It is worth to note, that the rpm of the engine is not constant, it fluctuates by around $300 \mathrm{rpm}$ (at idle). In case of this car, a one-cylinder engine is fitted into the chassis without a balancing shaft. Consequently, intense vibration is induced in the bodywork, body panels and on the aerodynamic parts, which increases the noise level. This extra noise is superimposed on the already loud exhaust noise. After reducing the dominant engine noise by the right mapping and with the properly designed exhaust system, further reduction can be achieved by the evaluation of modal analysis measurement for the structure. This can be done with the above-mentioned frame optimization and via appropriate design of the pickup points for the body panels and aerodynamic devices.

The purpose of this paper is to demonstrate the usefulness of modal analysis and to determine the NVH (Nosie, Vibration and Harshness) characteristics of a single-seater race car. After introducing the applicability of the modal analysis, the simulations and the measurements are presented. Finally, the results are critically evaluated and conclusions are drawn. The



first steps of this work were presented in a previously published conference paper [2]. The present article complements the previous work with more detailed description of theoretical background, measurement and evaluation process, and with additional simulations including sensitivity test.

\section{Determination of modal behaviour using FEM}

There are a lot of advantages to run simulations. First of all, the given component can be optimized from engineering acoustics, fatigue and comfort points of view. Furthermore, simulations are useful for selecting the accelerometer positions for experimental modal analysis. Not to mention, that they are necessary to validate the theoretical background of the experimental technique. In this way one can make sure that all the simulation and experimental settings were appropriate.

After determining the critical excitation frequency range of the engine, first the simulations had to be executed, taking into account the time- and cost-effectiveness. So as to predict the dynamic behaviour, the modes of the frame and to select the above-mentioned pickup points, Abaqus FE software was used.

The first simulation was performed both with shell and beam elements. Considering the resulted displacements, both solutions (using shell or beam elements) gave the same results. Note, that this is not true for the stresses in the structure caused by the loads. In Fig. 1 the first simulated flexural mode is presented.

Fig. 1 First simulated flexural mode using shell (left) and beam elements (right)

The main benefit of a "beam element method" is that it is less time-consuming, especially when considering the pre-processing part of the simulation. After investigating the effects of the element types, only beam elements were used in later studies. During the

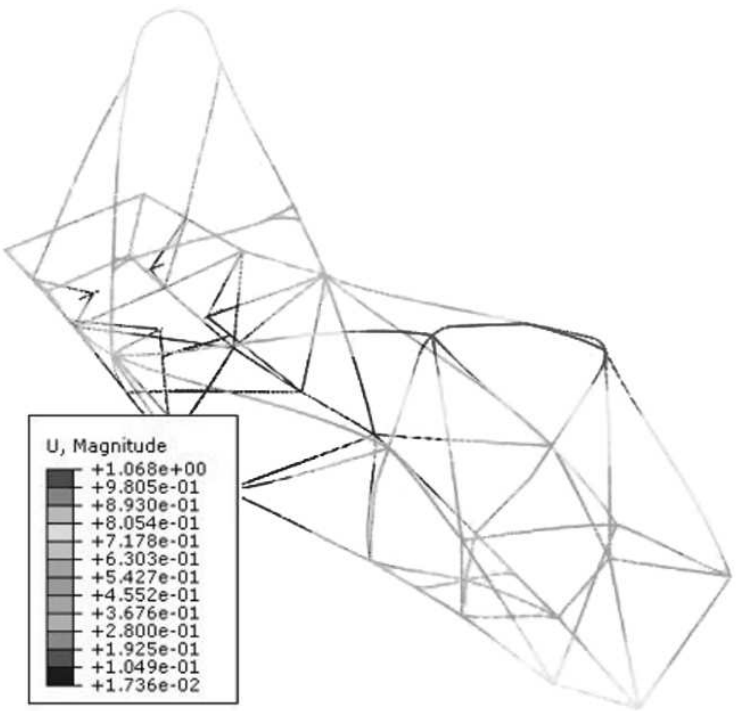

simulations the $0-1000 \mathrm{~Hz}$ rage was examined, despite the second engine order does not even reach 500 $\mathrm{Hz}$.

Tab. 1 shows the natural frequencies of the frame, corresponding to the first 10 flexural modes (modes 
1-6 corresponds to the rigid body vibrations). In the early phase of the design process, the first simulation was conducted with approximate material properties. The results of this can be seen in the second column of Tab. 1. Later on the simulation was carried out using the applied material's real mechanical properties. The results of that simulation using the real properties of the frame are shown in the third column of Tab. 1. One can conclude that an approximate error of $1.5 \%$ was resulted (regarding the natural frequencies) when inaccurate material properties were used.

In case of the $\mathrm{FE}$ simulation the eigenfrequencies (eigenvalues) and the eigenmodes (eigenvectors) are extracted from the simplified generalized equation of motion [3][4]:

Where:

$$
0=M \ddot{q}+K q
$$

$\mathbf{M}$ is the mass matrix [kg],

$\mathbf{K}$ is the stiffness matrix $[\mathrm{N} / \mathrm{m}]$, $\mathrm{q}$ is the displacement vector $[\mathrm{m}]$ and

$\ddot{\mathrm{q}}$ is the acceleration vector $\left[\mathrm{m} / \mathrm{s}^{2}\right]$.
This simplified equation of motion is used to describe undamped dynamic behaviour without real outer excitation. From this, it can be seen that the natural frequencies and corresponding modes are hugely effected and dependent on the mass matrix and on the stiffness matrix. These matrices include all the following information such as geometry, density, Young's modulus, which all together define the mass, mass distribution and the stiffness of the structure as well.

From this and from Tab.1, one can also see that the Young's modulus and material density - more exactly the ratio of them - has huge influence on mode density, namely on the number of modes in a given frequency range and in addition to this on the frequency of the first flexural mode. Note that, the geometry was not changed during the study. In order to conduct an FE simulation the Poisson's ratio has to be given, however it has negligible effect on the modal parameters. Material damping has to be considered during excited cases. It has significant effect on vibration response, on the structural damping. The structural damping is frequency dependent and it can be calculated from the resonance peaks of the FRFs using Half-Power Bandwidth method.

Tab. 1 Simulation results using different specific stiffness

\begin{tabular}{|c|c|c|}
\hline \multirow{2}{*}{ Flexural mode } & $\begin{array}{c}\text { Eigenfrequencies given by using } \\
\text { supposed properties [4] } \\
\varrho=7.83 \mathrm{~g} / \mathrm{cm}^{3} \\
\mathrm{E}=210 \mathrm{GPa}\end{array}$ & $\begin{array}{c}\text { Eigenfrequencies given by using } \\
\text { real properties } \\
\varrho=7.85 \mathrm{~g} / \mathrm{cm}^{3} \\
\mathrm{E}=205 \mathrm{GPa}\end{array}$ \\
\hline Mode 7 & $62.75 \mathrm{~Hz}$ & $61.99 \mathrm{~Hz}$ \\
\hline Mode 8 & $114.05 \mathrm{~Hz}$ & $112.58 \mathrm{~Hz}$ \\
\hline Mode 9 & $114.67 \mathrm{~Hz}$ & $113.20 \mathrm{~Hz}$ \\
\hline Mode 10 & $116.10 \mathrm{~Hz}$ & $114.57 \mathrm{~Hz}$ \\
\hline Mode 11 & $116.59 \mathrm{~Hz}$ & $115.09 \mathrm{~Hz}$ \\
\hline Mode 12 & $121.85 \mathrm{~Hz}$ & $120.37 \mathrm{~Hz}$ \\
\hline Mode 13 & $142.28 \mathrm{~Hz}$ & $140.48 \mathrm{~Hz}$ \\
\hline Mode 14 & $156.22 \mathrm{~Hz}$ & $154.24 \mathrm{~Hz}$ \\
\hline Mode 15 & $167.26 \mathrm{~Hz}$ & $165.11 \mathrm{~Hz}$ \\
\hline Mode 16 & $174.57 \mathrm{~Hz}$ & $172.31 \mathrm{~Hz}$ \\
\hline
\end{tabular}

The first six modes, which are the rigid body modes, are not shown in Tab.1. These modes come from the six DOF (Degree of Freedom) referring to the motion's freedom in the three-dimensional coordinate system (3 rotational and 3 translational freedom). In order to separate the rigid body modes and the flexural modes, a "free-free mounting" was applied both in practice and in FE simulations [5]. There were no constraints, excitations or loads defined during the simulations. For instance, in Fig. 2 the $12^{\text {th }}$ eigenmode ( $6^{\text {th }}$ without rigid body modes) of the chassis is depicted from the FEM simulation. The corresponding frequency is $120.37 \mathrm{~Hz}$, while the measured value for the same modeshape is $115.81 \mathrm{~Hz}$. In this case the shell-element FEM result is shown to demonstrate the flexible deformation of the rod elements.
However, as the resonance frequencies and -shapes does not differ significantly from the results of the beam element FEM model, the results of the latter will be presented in the following - making the comparison to the experimental results easier.

As another example, the $8^{\text {th }}$ eigenmode $\left(2^{\text {nd }}\right.$ without rigid body modes) of the frame from the experimental modal analysis can be seen in Fig. 3, where the corresponding frequencies in case of both the simulation and the measurement are around $113 \mathrm{~Hz}$. These eigenmodes are critical considering the wing vibration and the noise level regulations. During one of the noise pressure level measurements the engine speed has to be at least $6500 \mathrm{rpm}(108.33 \mathrm{~Hz})$ and one must consider on top of this the previously mentioned rpm fluctuation as well. As it can be seen on Fig. 2 and Fig. 
3, the highest displacements belong to the "main hoop" (the top-most part of the frame) and the "main hoop bracing" (the skew stiffening rods going backward from the top of the main hoop), where the wing's pickup points are located. This is why the wing vibrates quite intensely, and the noise level is increased at that engine speed. Not to mention the effectiveness of the aerodynamics, which is spoiled by the vibrations. It is worth mentioning furthermore, that the most commonly used frequency range, in which the engine operates during the race, is $6600-7000 \mathrm{rpm}$ $(110-116.7 \mathrm{~Hz})$.

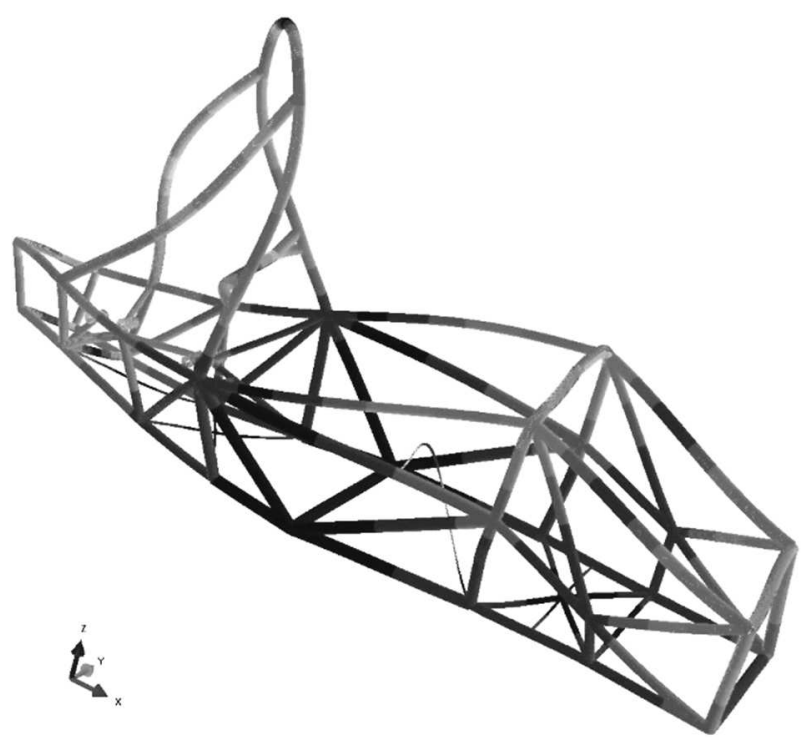

Fig. $212^{\text {th }}$ simulated eigenmode

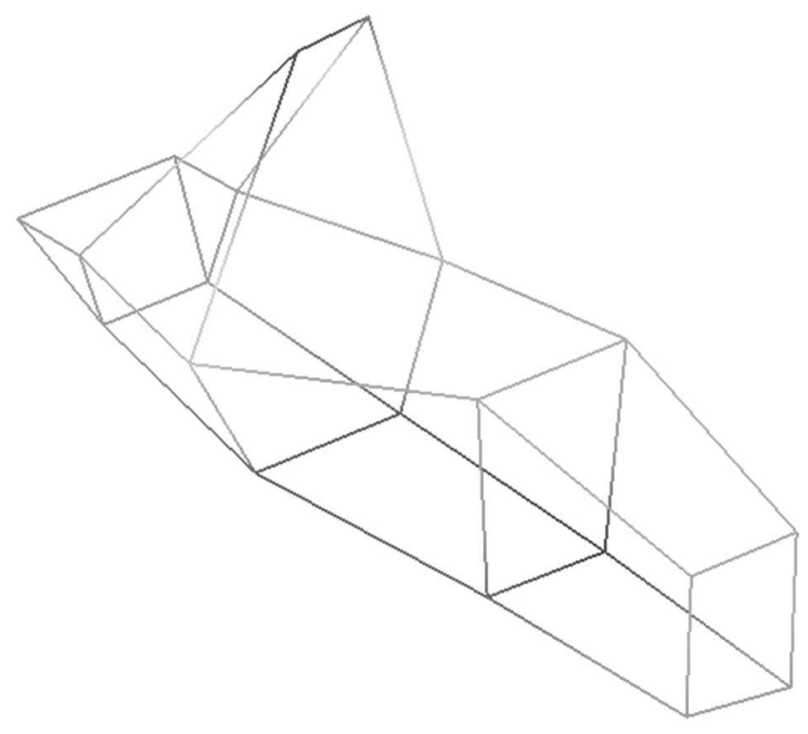

Fig. $38^{\text {th }}$ experimental eigenmode

The 7th natural frequency (simulated: $\sim 62 \mathrm{~Hz}$, measured: $\sim 56 \mathrm{~Hz}$ ) is close to the idle frequency of the engine $(\sim 50 \ldots 55 \mathrm{~Hz})$. In Fig. 4, the 7 th simulated eigenmode ( $\left(1^{\text {st }}\right.$ without rigid body modes $)$ can be seen, which heavily influences the chassis response to en- gine idle. This is one of the sources of the heavy vibrations besides idling.



Fig. $47^{\text {th }}$ simulated eigenmode

\section{Experimental modal analysis}

The determination of the accelerometer positions was based on FE simulations. During the sensor position selection the expected vibration shapes and required response functions have to be considered. According to that, two different practical ways of application can be chosen. In the first case, one can define both the local and global modes of the tubular frame. To reach this, accelerometers have to be placed not just close to the nodes of the frame but also between them, e.g. to the middle points of the tubes. The application of such high number of accelerometers could influence the results badly, because of the extra weight comes from the mass of the sensors. On the other hand, in this case the flexural vibration of each tube can be determined, and the results can be compared to the shell element FEM model (see Fig. 2). Considering the required extra work, the second, simpler possibility was chosen: In this case, only the global modes can be examined, by placing the accelerometers close to the nodes only. It needs less accelerometers, less work, and the main goal of the research - the determination of the vehicle's basic vibration behaviour can be achieved equally, as only nodes can be used as force input points and as the pickup points of the aero devices - according to the Formula Student rules [1].

Regarding the interpretation of the results, we have to note, that the measured accelerations are proportional to the displacements of the given point. During the measurement, bungees (elastic ropes) were used to suspend the chassis on a rigid frame, as shown in Fig. 5.

This mounting solution ensured the separation of 
the flexural and the rigid body modes. During the experimental modal analysis, one electromagnetic shaker, one impedance head and 20 pieces of triaxial accelerometers were used to evaluate the global modes. In the fifth picture 4 shakers can be seen, which were used during an independent previous measurement, which was an equivalent torsional stiffness measurement, however the mounting of the frame was the same. Note that in some cases it would be more reasonable to apply more excitation points in order not to miss any modes of the structure. This was a drawback of the selected experimental method. The impedance head was mounted to the tip of the stinger and it was glued to the excitation point (Fig. 6). With the help of an impedance head, both force and acceleration can be recorded. It is worth to note that a poorly chosen stinger can spoil the measured results. If the stinger is longer than it supposed to be, that can cause buckling phenomenon [6]. If it is too short, it generates stiffening effect to the structure, which can also impair the measured values. Therefore, the stinger's length was selected to be $100 \mathrm{~mm}$ beside a diameter of $3 \mathrm{~mm}[6][7][8]$.

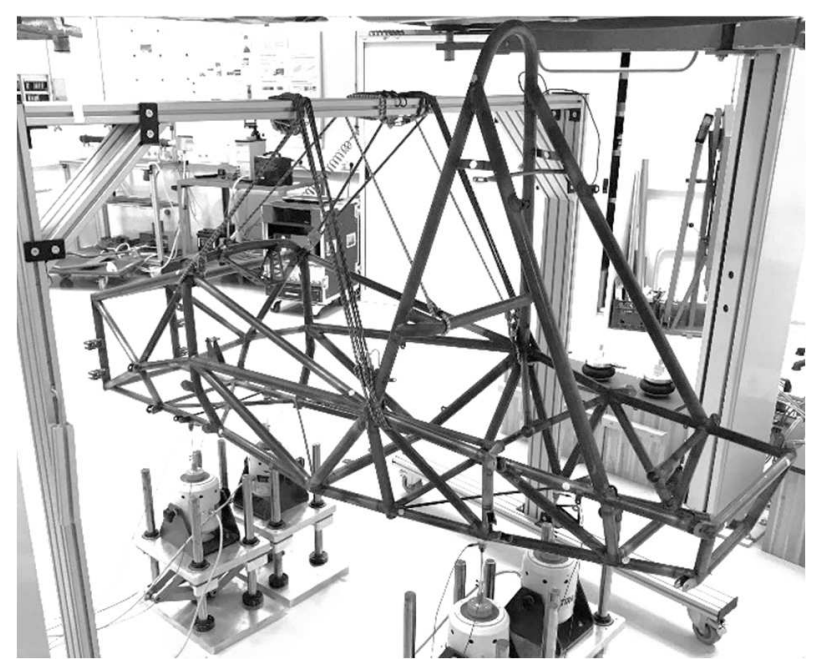

Fig. 5 Experimental NVH setup

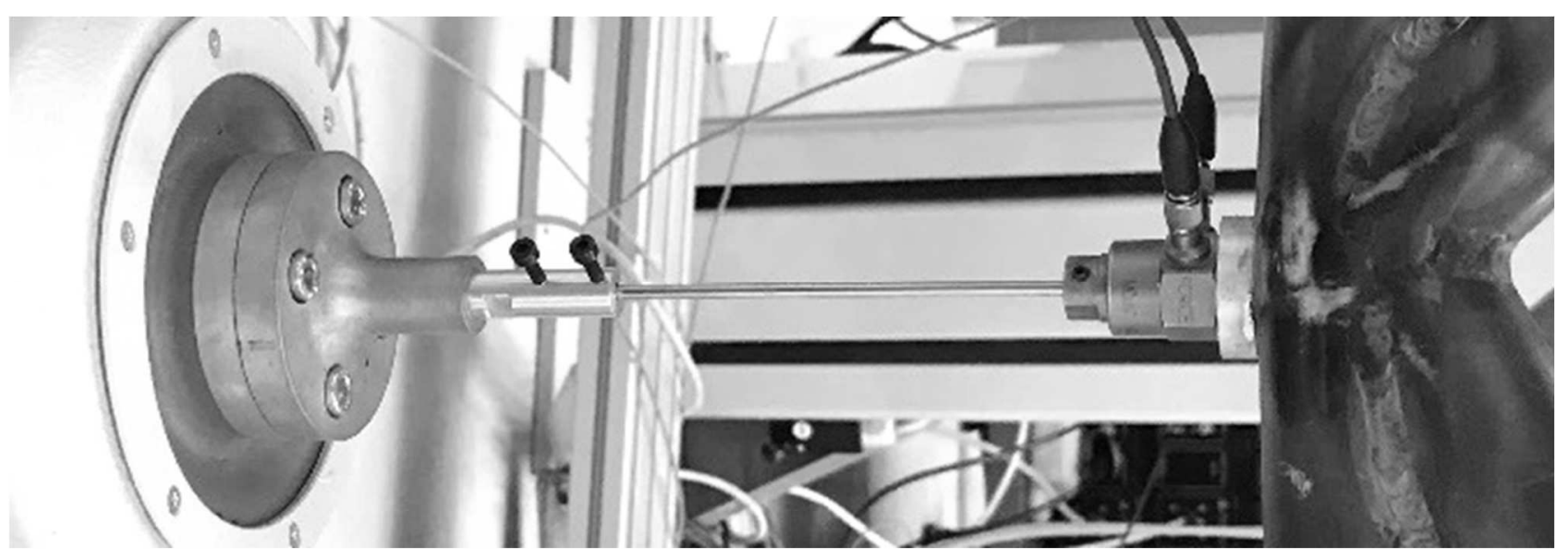

Fig. 6 The assembly of the excitation mechanism

Different excitation signals can be selected during the experimental work, here random white noise was used. With the help of the FFT (Fast Fourier Transformation) the recorded data were transformed from time domain to frequency domain [9]. From experimental point of view, one of the aims was to improve the coherence. The coherence can be visualized with a coherence curve along the frequency range, as it can be seen in Fig. 7. It can also be described with a single value, which can vary between 0 and 1 . The coherence describes the stability of the relation between the excitation and response functions measured by the accelerometers. If the value of the coherence is 1 , then it shows complete correlation between the two functions. The coherence is one of the indicators of the measurement properness, if any measurement error occurs (e.g. loosening of the stinger, movement of the shaker stand etc.), coherence curve will likely show it.
Two curves can be seen in Fig. 7, the curve marked with solid (red) line shows the coherence for one of the sensors after the very first measurement. In order to get reliable results that had to be improved. The dashed line shows the best coherence after finding the optimal setup. During the measurement the following aspects were considered to improve the coherence: excitation frequency range, excitation force, the type of the excitation signal, time/frequency resolution, number of bungees, isolation of unwanted contacts. Silicon isolations were used between the bungees and the frame for better results. "Nyquist stability criterion", a stability test was used to find the reasonable resolution and to eliminate measurement errors. Furthermore, the stiffness of the mounting was modified by applying higher number of bungees, to ensure the above mentioned appropriate "free-free mounting". 




Fig. 7 Evolution of the coherence according to measurements settings

Tab. 2 Comparison of simulation and measurement results

\begin{tabular}{|c|c|c|c|}
\hline Description of flexural modes & $\begin{array}{c}\text { Eigenfrequencies given by } \\
\text { simulation } \\
\text { (beam element FEM, } \\
\text { using real material proper- } \\
\text { ties: } \\
\mathrm{Q}=7.85 \mathrm{~g} / \mathrm{cm}^{3}, \mathrm{E}=205 \\
\mathrm{GPa}\end{array}$ & $\begin{array}{c}\text { Eigenfrequencies given } \\
\text { by measurement }\end{array}$ & $\begin{array}{c}\text { Deviation of simu- } \\
\text { lation from mea- } \\
\text { surement result }\end{array}$ \\
\hline Torsion (X-axis) - see Fig. 8 & $61.99 \mathrm{~Hz}$ & $55.81 \mathrm{~Hz}$ & $11.1 \%$ \\
\hline Bending (Y-axis) & $112.58 \mathrm{~Hz}$ & $113.60 \mathrm{~Hz}$ & $-0.9 \%$ \\
\hline Torsion (Main Hoop + X-axis) & $120.37 \mathrm{~Hz}$ & $115.81 \mathrm{~Hz}$ & $3.9 \%$ \\
\hline Intense Torsion (Main Hoop + X-axis) & $140.48 \mathrm{~Hz}$ & $143.00 \mathrm{~Hz}$ & $-1.8 \%$ \\
\hline Complex (Main Hoop Torsion + & $165.11 \mathrm{~Hz}$ & $153.12 \mathrm{~Hz}$ & $7.8 \%$ \\
\hline Shear) & $172.31 \mathrm{~Hz}$ & $177.17 \mathrm{~Hz}$ & $-2.7 \%$ \\
\hline Pulsation & $182.51 \mathrm{~Hz}$ & $189.74 \mathrm{~Hz}$ & $-3.8 \%$ \\
\hline Complex (Pitch + Pulsation) & $195.29 \mathrm{~Hz}$ & $191.38 \mathrm{~Hz}$ & $2.0 \%$ \\
\hline Yaw (Z-axis) - see Fig. 9 & \multicolumn{2}{|c|}{} \\
\hline
\end{tabular}

Tab. 2 compares the most important modes and the natural frequencies resulted by simulation (dynamic FEM with beam elements) and measurement. As it was mentioned before, first the simulation was conducted with supposed material properties in the early phase of the design process. Later on the simulation was carried out using the applied material's real mechanical properties, resulting higher natural frequencies by around $1.5 \%$. The eigenfrequencies according to this further improved simulation are shown in the second column of Tab. 2. Note that in case of the experimental results the accelerometers were considered as the nodes of the chassis, which were as close to the real nodes of the frame as possible. The comparison of some experimental- and simulation results can be seen in Fig. 8-9.

As a consequence, the main modes (listed in Tab. 2) are in good correlation regarding the simulation and experimental modal analysis results: modal shapes highly correlate to each other, and for 6 modes out of 8 the deviation of natural frequencies is within $4 \%$, while the largest deviation (in case of the first mode) is slightly above $10 \%$. This can evidently come from the manufacturing inaccuracies (wall thickness deviations, welding, etc.). 



Fig. 8 Flexural mode: Torsion around X-axis - Left: simulated (62 H₹); Right: measured (56 H₹)
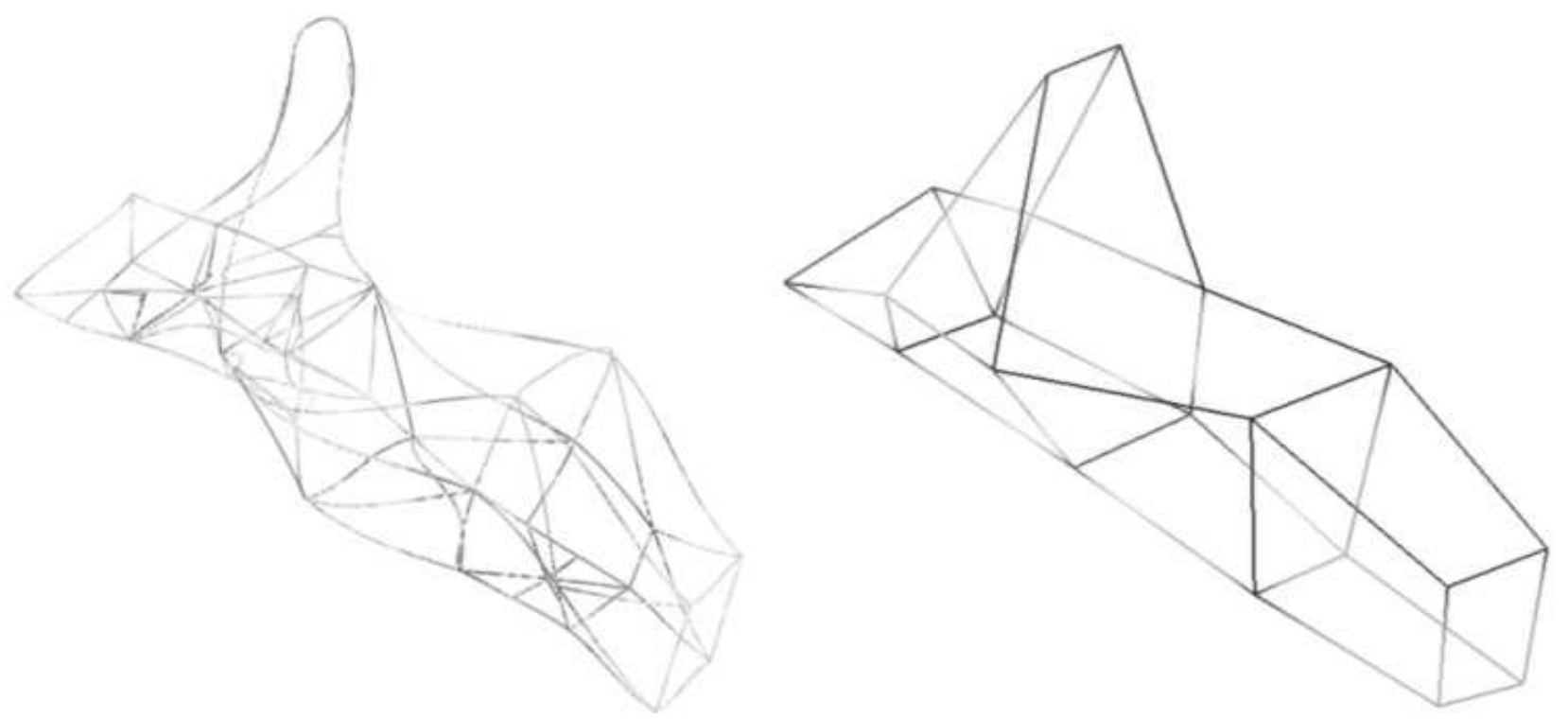

Fig. 9 Flexural mode: Yaw around Z-axis - Left: simulated (195 H₹); Right: measured (191 Hz)

\section{Conclusions and future plans}

During the research the main goal was to characterize the frame's dynamic mechanical behaviour by determining modal parameters of the chassis, aiming vibration-, noise- , sound pressure level- and failure probability reduction. Hence, the modal analysis of the chassis was carried out via simulation and experimental method. The main focuses of interest were the properness and accuracy of the investigations in order to get the results with the highest possible reliability, as modal analysis results can be easily spoiled by small inaccuracies.

The critical excitation source was found to be the engine. As a result, more resonant shapes were identified which can be excited by the engine, and which are critical from the point of view of noise, vibration and fatigue. As it was presented, the deformation amplitude of the chassis could be reduced by increased damping, as well as the modes and the corresponding frequencies could be easily de-tuned by the modification of the specific stiffness of the structure.

The dominant eigenmodes (resonance shapes and frequencies) were found both in the simulation and in the experimental modal analysis. The simulation and experimental results were in good agreement, differences can easily be explained by the manufacturing inaccuracies, such as welding, cutting and assembling errors. Due to the limited number of sensors, some modes from the simulation were not got from the experimental modal analysis - mainly the modes with high local impact (local tube vibrations). On the other 
hand, some theoretically symmetrical modes were recognized experimentally as two different modes (in contrast with the simulation), which comes also from the imperfect realization of the frame. Beside these smaller issues - which can be considered as normal anomalies when comparing the results of simulation and experimental dynamic analysis - the FEM simulation and its validation is considered to be successful and effective.

During the research, more suggestions for future developments were discovered, which are the followings: it would worth to put more emphasis on the excitation: using more shakers or impact hammer excitation (at multiple points) can ensure the more effective excitation of all modal vibrations. For better and more complete comparison between simulation and experimental results, MAC (Modal Assurance Criterion) could be used and the FRFs (Frequency Response Functions) of both solutions could be evaluated more deeply. Furthermore, the manufacturing inaccuracies could be quantified and more realistic FEM model could be generated and investigated by means of e.g. 3D scanning.

\section{Acknowledgement}

This paper was written with the support of the project titled "Internationalisation, initiatives to establish a new source of researchers and graduates, and development of knowledge and technological transfer as instruments of intelligent specialisations at Széchenyi István University" (project number: EFOP-3.6.1-16-2016-00017).

\section{References}

[1] Formula Student Rules (2019). Version: 1.1, Rev-713. Online: https://www.formulastudent. de/fsg/rules/\#c3938

[2] SCHWEIGHARDT, A., VEHOVSZKY, B., FESZTY, D. (2019). NVH and modal analysis in vehicle industry. In: Perner's Contact, Vol.
XIX, Special Issue 2, pp. 258 - 266. University of Pardubice. Czech Republic.

[3] HE, J., Fu, Z. (2001). Modal Analysis, pp. $94-$ 99. Butterworth-Heinemann, Oxford.

[4] DIŽO, J., HARUŠINEC, J., BLATNICKÝ, M. (2018). Computation of modal properties of two types of freight wagon bogie frames using the finite element method. In: Manufacturing Technology, Vol. 18, No. 2, pp. 208-214. J. E. Purkyne University in Usti n. Labem. Czech Republic.

[5] OKUMA, M., SHI, Q. (1997). Identification of principal rigid body modes under free-free boundary condition. In: Journal of Vibration and Acoustics, Vol. 119, No. 3, pp. 341-345. ASME. USA.

[6] VEHOVSZKY, B., GOÓR, D., FESZTY, D., GAZDAGH, Z., RÁBEL, A. (2018). Potential errors of acoustical testing induced by stinger excitation. In: Proceedings of the 25th International Congress on Sound and Vibration, Vol. 1, No. 8, pp. 304-314. Curran Associates. Hiroshima.

[7] PERES, M.A., BONO, R.W., BROWN, D.L. (2010). Practical aspects of shaker measurements for modal testing. In: Proceedings of ISMA, pp. 2539-2550. Leuven.

[8] ClOUTIER, D., AVITABILE P., BONO, R. W., PERES, M. A. (2009). Shaker/Stinger effects on measured frequency response functions. In: Proceedings of the XXVII IMAC Conference, pp. 197-203. UMASS Lowell. Orlando.

[9] CHEN, J., XU, Y. L. (2002). Identification of modal damping ratios of structures with closely spaced modal frequencies. In: Structural Engineering and Mechanics, Vol. 14, No. 4, pp. 417-434. Techno-Press. Korea. 